\title{
Effect of geometric aspect ratio and orifice length on the sound pressure level of a Helmholtz resonator
}

\author{
Mubarak Yakubu $^{a}{ }^{*}$, Isam Janajreh ${ }^{b}$ \\ ${ }^{a}$ Khalifa University, Abu Dhabi, United Arab Emirates \\ ${ }^{a}$ Khalifa University, Abu Dhabi, United Arab Emirates
}

\begin{abstract}
The acoustic performance of a typical Helmholtz resonator is important for optimizing sound absorption coefficient. The acoustic impedance of the Helmholtz resonator varies with different geometric features. As such, various studies have been conducted to investigate some parametric effects like neck length, extended neck, orifice size, cavity size and glazing flow speed. In this study, we explore, numerically, the effect of cavity shape and geometric aspect ratios among other features. We determined the optimum geometric shapes, dimension and flow condition for better sound absorption of the Helmholtz resonator.
\end{abstract}

Keywords: Helmholtz resonator, transmission loss, acoustic impedance, resonance frequency.

\section{Introduction}

Helmholtz resonator, once properly tuned, has strong sound attenuation characteristics, therefore, it is commonly employed as a noise attenuation device in ducted systems [1]. It has received a lot of attention in the control of low-frequency noise that propagates within the air conditioning duct channel in which the flow rates are usually small [2]. A proper design of such a resonator is important especially for low-frequency noise control in buildings where the conventional dissipative type silencers are not usually effective, thereby leading to a significant loss in static air pressure. The problems of lowfrequency duct noise has been tackled by the development of active control [3]. The robust performance and stability of the Helmholtz resonator makes it generally acceptable in the noise control industry.

The importance of the Helmholtz resonator has called the attention of many design engineers and researchers. Recently, several extensive studies have been undertaken to predict and improve the transmission loss of resonators [4], [5]. Many different arrangements and complicated geometries have been considered. For instance, Chanaud [6] explored the effects of different shapes of orifice, Dicky and Selamet [7] studied the effects of cavity aspect ratio, Selamet and Lee [8] proposed extended neck into the resonator cavity and Griffin et al. [9] suggested coupling two resonators. The idea of coupling dissipative silencers with Helmholtz resonator has also been proposed [10]. Although several suggestions have been made, however, some of these suggestions appear complex or are impractical. The aero-acoustic effect of the Helmholtz resonator has also been investigated [11].

The acoustic impedance at the outlet of the Helmholtz resonator has a significant effect on the obtainable sound absorption coefficient. The highest transmission loss or sound absorption coefficient takes place at the resonance frequency of the resonator and the extent of the effect highly depends on the acoustic impedance. The low-frequency plane wave theory suggests that a reduction of the resistive effect not only results in a high

* Corresponding author. Tel.: +9712312 3286

Fax: +971 2312 3286; E-mail: $100060013 @$ ku.ac.ae

(C) 2021 International Association for Sharing Knowledge and Sustainability

DOI: 10.5383 /ijtee.17.02.003

sound transmission loss but also only results to an increased in sound absorption coefficient. with a resistance higher than that of the medium. Tapering the neck of a resonator can improve its performance since a gradual change towards the cavity from the neck will have a lower flow resistance. However, this may affect the resonance frequency of the Helmholtz resonator.

(a)

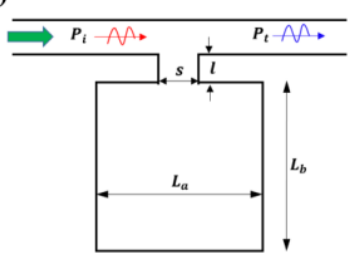

Figure 1: Schematic illustration of the Helmholtz resonators geometric features (a) For Rectangular cavity (b) For Circular cavity

In this work, the transmission power of a typical Helmholtz resonator is studied numerically. A $2 \mathrm{D}$ model is developed which represents a cavity connected to a duct via a neck of negligible length. Incident plane waves propagate through the duct. The Helmholtz resonator system consists of several features of which five were investigated, these include: 1) the neck length, 2) the orifice length, 3) the cavity shape, 4) the cavity size, and 5) the cavity aspect ratio. In Sect. 2, the numerical model and governing equations are described. The model is validated with an experimental study found in the literature. In Sect. 3, the effect of the geometric features of the Helmholtz resonator is studied under a frequency range of 0 to $2000 \mathrm{~Hz}$ and the key findings are summarized in Sect. 4.

\section{Model development}

In the present study, we consider a Helmholtz resonator with circular and rectangular shaped cavities as shown in Fig. 1. The geometric features of the resonator are varied, and the effect is studied. The mean grazing flow with the duct of the resonator is considered negligible, i.e., the flow is simulated in stationary air. 
The problem is solved using the incompressible Navier-Stokes equations.

\subsection{The governing equations}

The governing equations include the mass conservation equation, linearized momentum equation for incompressible viscous flow, and the energy equation given in Cartesian coordinate.

\section{The mass conservation equation}

$$
\frac{\partial \rho}{\partial t}+\nabla \cdot\left(\rho_{0} \vec{u}+\rho \vec{u}_{0}\right)=S_{l s}
$$

Where, $S_{l s}$ is the acoustic perturbation generated from the wave source. It is simulated as a monopole generated from a sinusoidal perturbation source $S_{l s}=\sum_{n=1}^{N} \lambda_{n} \sin \left(\omega_{n} t\right)$. The perturbation is given as pressure, velocity, temperature and density denoted as $p, \vec{u}, T, \rho$. The subscript 0 means steady-state flow parameter

\section{The momentum equation}

$$
\rho_{0}\left[\frac{\partial \vec{u}}{\partial t}+\left(\vec{u}_{0} \cdot \nabla\right) \vec{u}\right]+\rho\left(\vec{u}_{0} \cdot \nabla\right) \vec{u}_{0}=\nabla \cdot \sigma-\vec{u}_{0} S_{t s}
$$

\section{The energy equation}

$$
\begin{aligned}
\rho_{0} c_{p}\left[\frac{\partial T}{\partial t}+\left(\vec{u} \cdot \nabla T_{0}\right)\right. & \left.+\vec{u}_{0} \cdot \nabla T\right]+\rho c_{p}\left(\vec{u}_{0} \cdot \nabla T_{0}\right) \\
& -\alpha_{p} T_{0}\left[\frac{\partial p}{\partial t}+\vec{u} \cdot \nabla p_{0}+\vec{u}_{0} \cdot \nabla p\right] \\
& -\left(\alpha_{p} T\right) \vec{u}_{0} \cdot \nabla p_{0}=\nabla \cdot(k \nabla T)+\Phi
\end{aligned}
$$

Where $\Phi$ is the viscous dissipation function. The equation of state is $p=\rho R T$.

The conservative stress tensor equation and the linearized equation of state are given as:

$$
\begin{gathered}
\sigma=-p \mathbf{I}+\tau=-p+\mu\left[\nabla \vec{u}+(\nabla \vec{u})^{T}+\left(\mu_{\beta}\right.\right. \\
\left.\left.-\frac{2}{3} \mu\right)(\nabla \cdot \vec{u}) \mathbf{I}\right]
\end{gathered}
$$

Where, $\rho=\rho_{0}\left(\beta_{T} p-\alpha_{p} T\right) \cdot \tau$ is the viscous stress, $\mu$ is the dynamic viscosity, $\mu_{\beta}$ is the bulk viscosity, $\mathbf{I}$ is the identity matrix. The time derivative $\partial X / \partial t$ of a given parameter in the frequency domain can be replaced by multiplication with $j \omega X$

The term for the fluctuation velocity corresponding to the Reynolds stresses results from turbulent motion. From various turbulent flow models, $k-\varepsilon$ model is adopted in this study, to determine that the turbulent kinetic energy and rate of dissipation. The $k-\varepsilon$ turbulence model is given for turbulence kinetic energy $k$ as [12]:

$$
\begin{aligned}
\frac{\partial(\rho k)}{\partial t}+\frac{\partial\left(\rho k u_{i}\right)}{\partial x_{i}}= & \frac{\partial}{\partial x_{j}}\left(\left(\mu+\frac{\mu_{t}}{\sigma_{k}}\right) \frac{\partial k}{\partial x_{j}}\right)+\mu_{t} G_{k} \\
& -\rho \varepsilon-\frac{2}{3}\left(\mu_{t} \frac{\partial u_{i}}{\partial x_{i}}+\rho k\right)+\mu_{t} G_{b}
\end{aligned}
$$

Where $\varepsilon$ is the rate of dissipation of turbulent kinetic energy given Eq. (6)

$$
\begin{aligned}
\frac{\partial(\rho \varepsilon)}{\partial t}+\frac{\partial\left(\rho \varepsilon u_{i}\right)}{\partial x_{j}}= & \frac{\partial}{\partial x_{j}}\left(\left[\mu+\frac{\mu_{t}}{\sigma_{\varepsilon}}\right] \frac{\partial \varepsilon}{\partial x_{j}}\right) \\
& +C_{1 \varepsilon} \frac{\varepsilon}{k}\left(\mu_{t}\left[G_{k}+C_{3 \varepsilon} G_{b}\right]\right. \\
& \left.-\frac{2}{3}\left[\mu_{t} \frac{\partial u_{j}}{\partial x_{j}}+k \rho\right] \frac{\partial u_{i}}{\partial x_{i}}\right)
\end{aligned}
$$

Where, $G_{k}$ and $G_{b}$ are the generation of turbulence kinetic energy due to mean velocity gradient and buoyancy, respectively.

\subsection{Model validation}

The linearized Navier-Stokes equation with $k-\varepsilon$ model is solved by the COMSOL MULTIPHYSICS using UMF Direct solver based on the Finite element method. The validation study is conducted on a 2D Helmholtz resonator with a cylindrical duct as shown in Fig. 2.

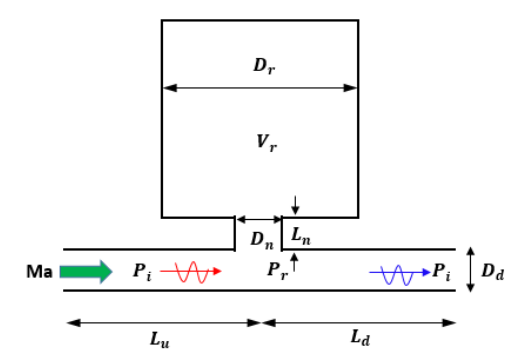

Figure 2: The schematic representation of the validation

\begin{tabular}{llll}
\multicolumn{4}{l}{ Table 1: Dimensions and flow condition of the validation model } \\
\hline Parameters & Values & Parameters & Values \\
\hline$L_{u}$ & $65.0 \mathrm{~cm}$ & $V_{r}$ & $2250 \rightarrow 4500 \mathrm{~cm}^{3}$ \\
$L_{d}$ & $65.0 \mathrm{~cm}$ & Ma & $0 \rightarrow 0.1$ \\
$D_{d}$ & $4.86 \mathrm{~cm}$ & $\rho_{0}$ & $1.2 \mathrm{~kg} / \mathrm{m}^{3}$ \\
$L_{n}$ & $1 \rightarrow 8.05 \mathrm{~cm}$ & $\mathrm{P}_{0}$ & $101325 \mathrm{~Pa}$ \\
$D_{n}$ & $4.04 \rightarrow 8.08 \mathrm{~cm}$ & $\omega / 2 \pi$ & $50-500 \mathrm{~Hz}$ \\
$D_{r}$ & $15.32 \mathrm{~cm}$ & $\mathrm{~T}_{0}$ & $297 \mathrm{~K}$ \\
$H_{r}$ & $24.42 \mathrm{~cm}$ & Mab & $0 \rightarrow 0.03$ \\
\hline
\end{tabular}

The flow conditions as well as the dimension of the Helmholtz resonator is determined from a study [13] and given in Table 1. In the validation study, the turbulence model is selected due to the presence of grazing flow [14]. The numerical model is compared with the experimental results obtained by Selamet et al. on the same dimensions and flow conditions [15]. The frequency range of $50-200 \mathrm{~Hz}$ is used for consistency with the experimental test.

The present numerical results are compared with that of Gang et al. [13] and the experimental results of Selamet et al. [15] The simulation is conducted with a time step of $1 \times 10^{-4} \mathrm{~s}$.

The plane waves are assumed to propagate through the duct from inlet to exit. Both the inlet and exit are assumed to perfectly match the layer boundary. The transmission loss (TL) of the Helmholtz resonator is given by the relation:

$$
T_{L}=10 \log _{10}\left(\left\|\frac{p_{i}(\omega)}{p_{t}(\omega)}\right\|^{2}\right)
$$

Where $p_{i}$ denotes incident acoustic waves at the upstream of the duct and $p_{t}$ is the transmitted acoustic wave at the down scream of the duct. 


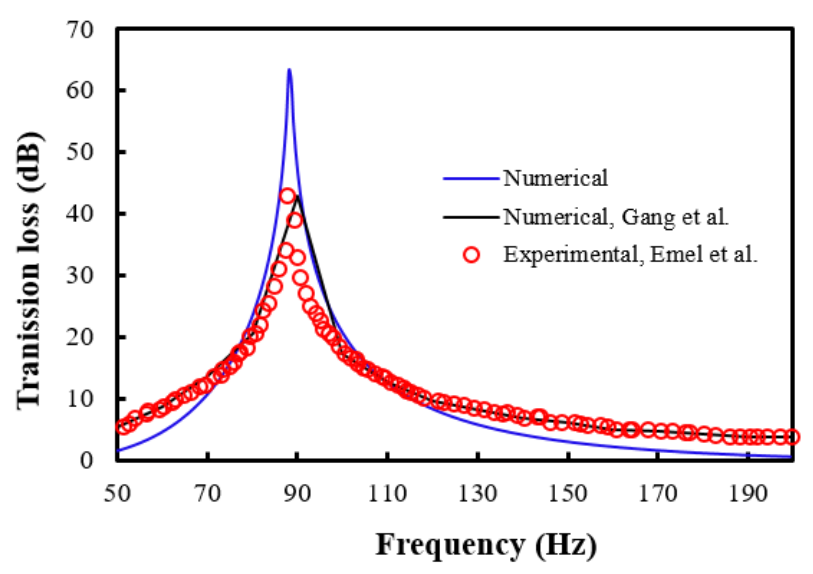

Figure 3: Validation of the developed model with experimental and numerical studies found in the literature.

\section{Results and discussion}

The validation model in Fig. 3 shows that numerical settings in commercial code, COMSOL MULTIPHYSICS, can predict the performance of the HR with a reasonable level of accuracy. Hence, the present model is implemented under the same study type and physics.

The geometries selected in this study and the flow conditions are illustrated in Fig. 5 and the parameters are given in Table 2.
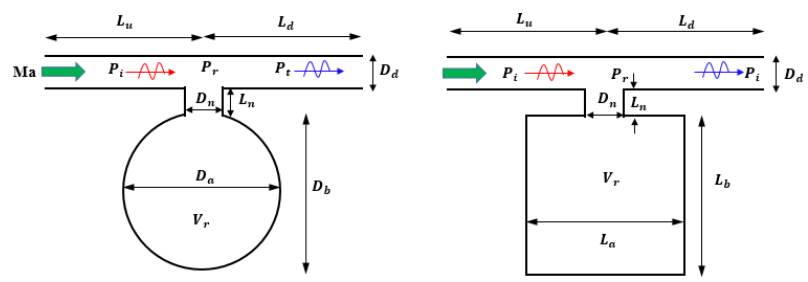

Figure 4: Illustration of the present model

Table 2: Dimensions and the flow condition of the present model.

\begin{tabular}{lll}
\hline Parameters & Values & Unit \\
\hline$V_{r}$ & $1600,2500,3600$ & $\mathrm{~mm}^{3}$ \\
$L_{u}$ & 50 & $\mathrm{~mm}$ \\
$L_{d}$ & 50 & $\mathrm{~mm}$ \\
$L_{n}$ & $1,10,20,30$ & $\mathrm{~mm}$ \\
$D_{d}$ & 10 & $\mathrm{~mm}$ \\
$D_{n}$ & $1,5,10,20$ & $\mathrm{~mm}^{2}$ \\
Medium fluid & Air & - \\
Shapes & Rectangular, Circular & - \\
Aspect & $0.5,1,2$ & - \\
\hline
\end{tabular}

\subsection{Mesh independence test}

A Mesh dependency test was conducted on the 2D Helmholtz resonator, and a triangular mesh is adopted, with grid elements sizes as shown in Fig. 6. The present model, having the rectangular shape cavity, is tested for the effect of mesh element size on the TL. Under a frequency range of $0-2000 \mathrm{~Hz}$, the result shows that the TL slightly changes with the mesh element size and attains a convergence at about 520 element size. The resonance frequency remains constant for all the sizes of the mesh as shown in Fig. 6.

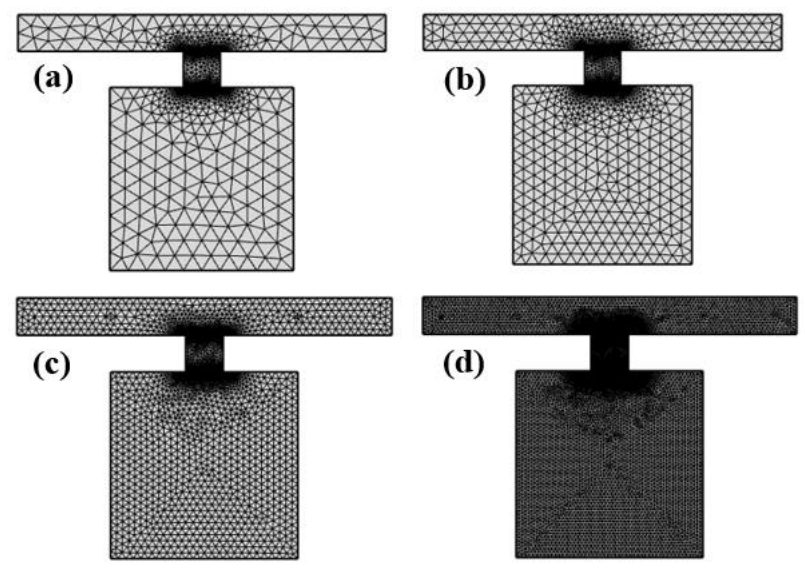

Figure 5: Numerical triangular mesh on the 2D model with difference mesh element sizes: (a) 384, (b) 520, (c) 642, and (d) 1254 .

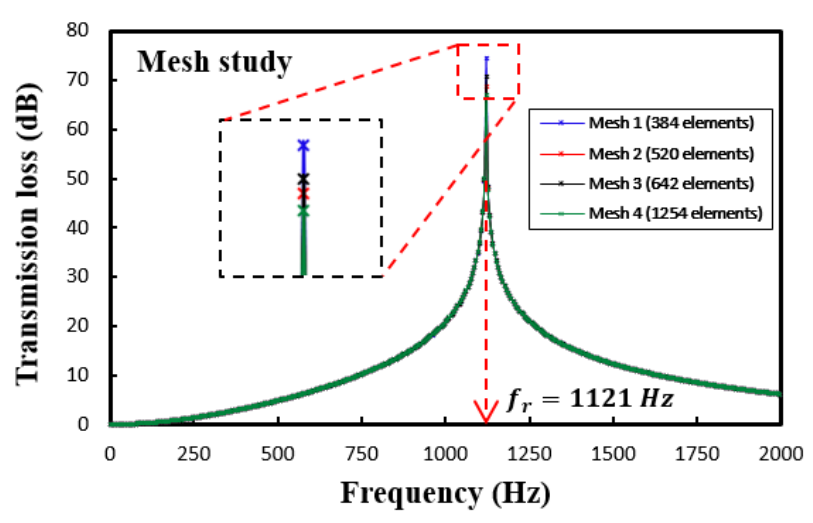

Figure 6: Transmission loss for different mesh element size

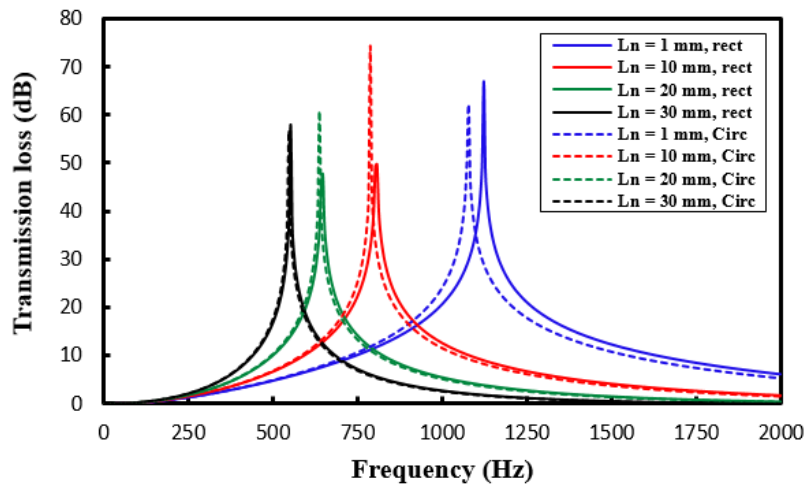

Figure 7: Effect of neck length on the transmission loss for different length of the neck 


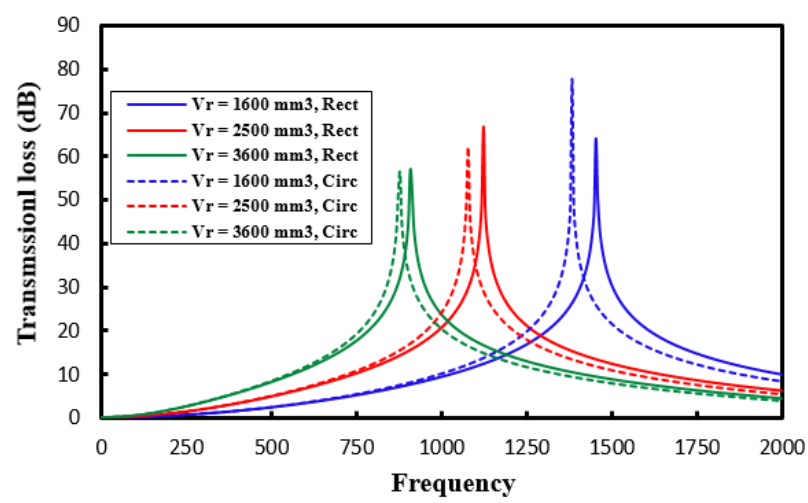

Figure 8: Effect of cavity volume on the transmission loss

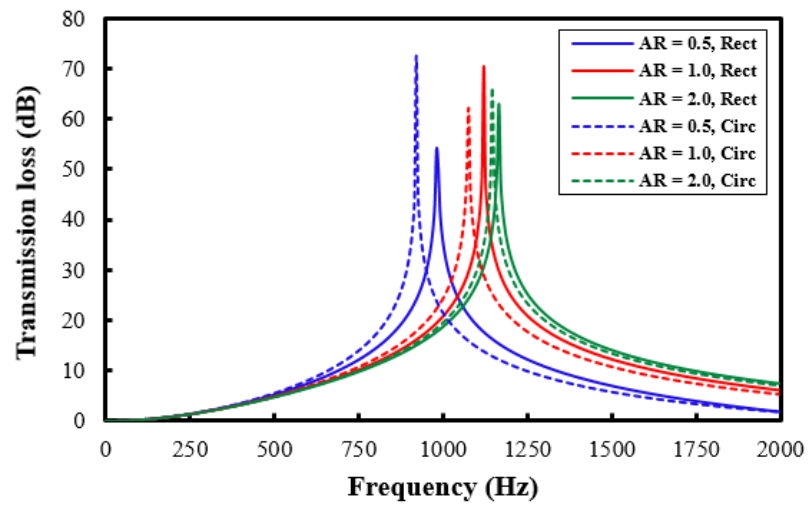

Figure 9: Effect of cavity Aspect ratio on the transmission loss

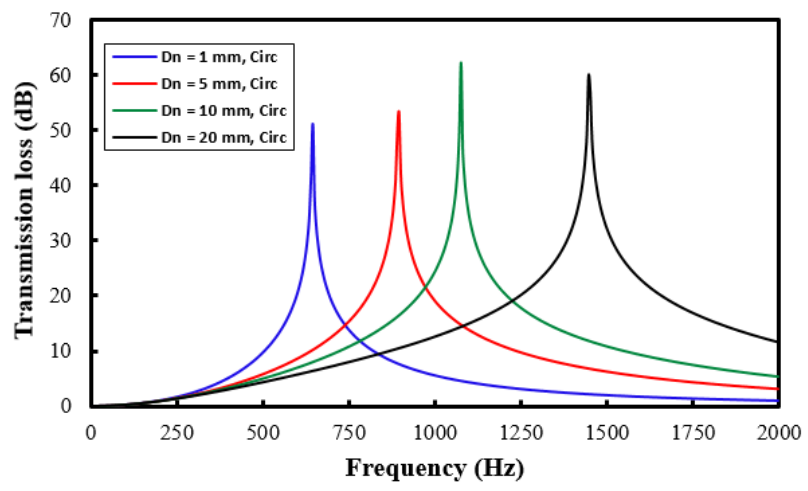

Figure 10: Effect of orifice length on the transmission loss

The effect of various geometric parameters is investigated. Fig. 7 shows that, for rectangular cavity shape, the TL decreases with an increase in neck length until a certain minimum TL which occurs at about $10 \mathrm{~mm}$. However, this effect is opposite in the circular shape cavity and the maximum TL occurs at $10 \mathrm{~mm}$. Although the cavity volume remains constant, the volume to surface area ratio is not the same for the two shapes. Therefore, this accounts for the counter-effect.

Fig. 8. shows how the cavity size affects the TL. The sensitivity of cavity size to TL is higher for the circular cavity. Also, the TL reduces with cavity volume linearly but in the rectangular shape, the sensitivity of TL becomes very low for lower cavity volumes.

As shown in Fig. 9 the Aspect ratio (AR) of the cavity cannot provide a wider range of resonance frequency but an inverse relation is found for the two shapes selected in the present study.
At $\mathrm{AR}=1$, the $\mathrm{TL}$ is highest in the rectangular cavity while having the lowest in the circular cavity.

In Fig. 10, considering only the circular cavity, the orifice length of HR is investigated. The TL increases with orifice length until a certain optimum value above which the TL begins to reduce. In this study, it was seen that an Orifice length of $10 \mathrm{~mm}$ has the highest TL.

\section{Conclusion}

The acoustic properties of an HR of varying cavity shape, size, aspect ratios, neck length, and orifice length are studied numerically. The HR operates in monotone and serves as an excellent absorber for flow with frequency same as its natural frequency.

The transmission loss (TL) of the HR serves as the metric for performance measurement. Various parameters that affect the performance of the HR are varied and the present study covers an assessment of the best configuration for the HR because a proper prediction of the TL will enhance the resonator's application range in the desired operating frequency.

Considering the shape effect of the HR, the numerical simulation shows that the cavity shape has an interactive effect with other geometric parameters. The HR with circular shape cavity has maximum $\mathrm{TL}$ at a lower resonance frequency than the rectangular shape cavity. TL increases steadily with neck length for rectangular shape cavity unlike in circular cavity where the TL increases in a parabolic shape with neck length until a certain optimum value $(10 \mathrm{~mm})$ before dropping to lower TL. The orifice length of $10 \mathrm{~mm}$ is the optimum in the present study. The optimum parameters can be utilized in design an HR with high sound absorption property.

The shape effect of the HR can be formulated analytically, and the TL can be optimized based on the desired application and frequency range.

\section{Acknowledgments}

The authors wish to appreciate the support of Khalifa University of Science, Technology and Research for providing the required funding and access to facilities to ensure the success of this research work.

\section{References}

Chen KT, Chen YH, Lin KY, Weng CC. The improvement on the transmission loss of a duct by adding Helmholtz resonators. Appl Acoust 1998;54:71-82. https://doi.org/10.1016/S0003682X(97)00036-4.

[2] Or KH, Putra A, Selamat MZ. Oil palm empty fruit bunch fibres as sustainable acoustic absorber. Appl Acoust 2017;119:9-16 https://doi.org/10.1016/j.apacoust.2016.12.002.

[3] Tang SK, Cheng JSF. On the application of active noise control in an open end rectangular duct with and without flow. Appl Acoust 1998;53:193-210. https://doi.org/10.1016/s0003-682x(97)00017-0.

[4] Ingard U. On the Theory and Design of Acoustic Resonators The Journal of the Acoustical Society of America. J Acoust Soc Am 1953;25:1037-61.

[5] Hersh AS, Walker BE, Celano J. Helmholtz resonator impedance model, part 1: nonlinear behavior. AIAA J 2003;41:795-808. 
[6] Chanaud RC. Effects of geometry on the resonance frequency of Helmholtz resonators. J Sound Vib 1994;178:337-48. https://doi.org/10.1006/jsvi.1994.1490.

[7] L / D 1 1996;i:512-7.

[8] Selamet A, Lee I. Helmholtz resonator with extended neck. J Acoust Soc Am 2003;113:1975-85. https://doi.org/10.1121/1.1558379.

[9] Selamet A, Lee IJ, Huff NT. Acoustic attenuation of hybrid silencers. J Sound Vib 2003;262:509-27. https://doi.org/10.1016/S0022-460X(03)00109-3.

[10] Tang SK. On Helmholtz resonators with tapered necks. J Sound Vib 2005;279:1085-96. https://doi.org/10.1016/j.jsv.2003.11.032.

[11] Meissner M. Excitation of Helmholtz resonator by grazing air flow [3]. J Sound Vib 2002;256:382-8. https://doi.org/10.1006/jsvi.2001.4219.

[12] Chen S, Zhao D. Numerical study of non-reacting flowfields of a swirling trapped vortex ramjet combustor. Aerosp Sci Technol 2018;74:81-92. https://doi.org/10.1016/j.ast.2018.01.006.

[13] Wu G, Lu Z, Xu X, Pan W, Wu W, Li J, et al. Numerical investigation of aeroacoustics damping performance of a Helmholtz resonator: Effects of geometry, grazing and bias flow. Aerosp Sci Technol 2019;86:191-203.

https://doi.org/10.1016/j.ast.2019.01.007.

[14] Pan W, Xu X, Li J, Guan Y. Acoustic damping performance of coupled Helmholtz resonators with a sharable perforated sidewall in the presence of grazing flow. Aerosp Sci Technol 2020;99:105573. https://doi.org/10.1016/j.ast.2019.105573.

[15] Selamet E, Selamet A, Iqbal A, Kim H. Effect of flow on helmholtz resonator acoustics: A three-dimensional computational study vs. experiments. SAE Tech Pap 2011. https://doi.org/10.4271/2011-01-1521. 